\title{
Evaluation of a standardized protocol for thrombin generation using the calibrated automated thrombogram : A Nordic study
}

\section{Ljungkvist, Marcus}

2019-03

Ljungkvist, M , Strandberg , K, Berntorp , E , Chaireti , R , Holme , P A , Larsen , O H , Lassila , R , Jouppila , A , Szanto , T \& Zetterberg , E 2019 , ' Evaluation of a standardized protocol for thrombin generation using the calibrated automated thrombogram : A Nordic study ' , Haemophilia , vol. 25 , no. 2 , pp. 334-342 . https://doi.org/10.1111/hae.13640

http://hdl.handle.net/10138/314537

https://doi.org/10.1111/hae.13640

publishedVersion

Downloaded from Helda, University of Helsinki institutional repository.

This is an electronic reprint of the original article.

This reprint may differ from the original in pagination and typographic detail.

Please cite the original version. 


\title{
Evaluation of a standardized protocol for thrombin generation using the calibrated automated thrombogram: A Nordic study
}

\author{
Marcus Ljungkvist $^{1}$ (D) | Karin Strandberg ${ }^{2}$ | Erik Berntorp $^{1}$ (D) | Roza Chaireti ${ }^{3,4}$ (D) \\ Pål André Holme ${ }^{5}$ | Ole Halfdan Larsen ${ }^{6}$ | Riitta Lassila ${ }^{7}$ | Annukka Jouppila ${ }^{8}$ | \\ Timea Szanto ${ }^{9}$ (D) | Eva Zetterberg ${ }^{1}$
}

${ }^{1}$ Department of Translational Medicine \& Centre for Thrombosis and Haemostasis, Lund University, Malmö, Sweden

${ }^{2}$ Clinical Chemistry, University and Regional Laboratories Region Scania, Malmö, Sweden

${ }^{3}$ Department of Hematology, Karolinska University Hospital, Stockholm, Sweden

${ }^{4}$ Department of Molecular Medicine and Surgery, Karolinska Institutet, Stockholm, Sweden

${ }^{5}$ Department of Haematology, Oslo University Hospital and Institute of Clinical Medicine, University of Oslo, Oslo, Norway

${ }^{6}$ Centre for Haemophilia and

Thrombosis, Aarhus University Hospital, Skejby, Denmark

${ }^{7}$ Unit of Coagulation Disorders, Department of Hematology, Helsinki University Central Hospital, Helsinki, Finland

${ }^{8}$ Helsinki University Central Hospital Research Institute, Helsinki, Finland

${ }^{9}$ Unit of Coagulation Disorders, HUSLAB Laboratory Services, Department of Clinical Chemistry, Helsinki University Hospital, Helsinki, Finland

\section{Correspondence}

Marcus Ljungkvist, Wallenberg Laboratory pl 2, University Hospital, Malmö, Sweden. Email: marcus.ljungkvist@med.lu.se

\section{Funding information}

Lund University Medical Faculty Foundation; Region Skåne; Baxalta; Lund University
Introduction: The thrombin generation assay-calibrated automated thrombogram (TGA-CAT) method is used to measure the overall coagulation capacity in plasma. However, the method is still considered to be a research tool, mainly because of its' lack of standardization.

Aim: Our study aimed to further raise the standardization level for the TGA-CAT method by evaluating a detailed standardization protocol and three reference plasmas' (RP)s ability to normalize results.

Methods: Six Nordic centres participated in the study, and with input from all centres a detailed laboratory standardization protocol based on the TGA-CAT manual of the manufacturer was established. Three types of plasma, hypo-,normal and hypercoagulable plasma were assessed. Three commercial lyophilized RPs were used for normalization of data. All samples were aliquoted at the Malmö centre and sent frozen at $-20^{\circ} \mathrm{C}$ to participating centres.

Results: Before normalization, all results under all testing conditions showed interlaboratory coefficient of variability of $10 \%$ or lower except for endogenous thrombin potential (12\%) and peak (14\%) in hypo-plasma with $1 \mathrm{pmol} / \mathrm{L}$ tissue factor as starting agent. Successful normalization, improving variability in results, was obtained with two of the three evaluated RPs (HemosIL RP and Affinity RP).

Conclusion: With our standardization concept, we were able to produce TGA-CAT results as robust as standard coagulation assays used in the routine laboratories. Normalization with HemosIL RP may be considered in populations with low or unknown coagulability, while when analysing plasma samples from populations where hypercoagulability is known or suspected, normalization with Affinity RP may be preferred.

\section{KEYWORDS}

haemophilia, normalization, reference plasma, standardization, test of agreement, thrombin generation assay 


\section{1 | INTRODUCTION}

In a number of studies, the thrombin generation assay-calibrated automated thrombogram (TGA-CAT) method has proven its usefulness for multiple purposes including diagnosis and management of bleeding disorders, ${ }^{1,2}$ detecting hypercoagulability ${ }^{3,4}$ and monitoring and characterization of oral anticoagulant drugs. ${ }^{5,6}$ However, most of these investigations are single-centre, and large prospective multi-centre studies are needed to further validate the results. Multi-centre studies have proven difficult to undertake due to a lack of standardization of the method. In recent years, several articles have addressed the problem of large inter-laboratory variability for the TGA-CAT method by presenting detailed standardization protocols. Focus has been placed both on analytical and pre-analytical factors ${ }^{7,8}$ as well as the use of reference plasmas (RP) ${ }^{10,11}$ for normalization of results (ie, dividing all values with the result of a common reference plasma) in the pursuit of an acceptable inter-laboratory variability for the method. ${ }^{12}$

With the aim to further improve the level of standardization for the TGA-CAT method, we evaluated the use of a detailed standardization protocol and normalization of data with a number of RP to reduce the inter-laboratory variability between six centres.

\section{2 | MATERIALS AND METHODS}

\section{1 | Study design}

The study was approved by the Regional Ethics Committee of Lund University, Sweden (Registry number: 2016/451). Written informed consent was obtained from all participants prior to the study.

The investigation consisted of two phases: First, a pre-study onsite inspection, and second, the implementation. In the pre-study inspection, all participating laboratories were evaluated by the same study coordinator. During the visit, all equipment and materials were inspected, that is, calibration of pipettes, software version (V5.0.0.742), and service protocol of the Fluoroskan Ascent analyzer (Thermo Scientific, Shanghai, China). Measurements of the instrument analysing temperature and room temperature (RT) were made. The local operator's and the inspector's TGA-CAT results were compared in a test run, that was performed to investigate the operator influence on the method variability.
In the implementation phase, all samples were run with two different initiating agents: Platelet-poor plasma (PPP)-reagent LOW (tissue factor [TF] concentration $1 \mathrm{pmol} / \mathrm{L}$ ) and PPP-reagent (TF concentration $5 \mathrm{pmol} / \mathrm{L}$ ). With the goal of covering the entire measuring range of the TGA-CAT method, three plasma types were assessed (hypo-, normal and hypercoagulable plasma). The hypo-plasma was a pool from four persons with severe haemophilia A treated at the Malmö Centre for Thrombosis and Haemostasis (MCTH). The normal plasma group consisted of four healthy volunteers having endogenous thrombin potential (ETP) values close to the mean value of the TGA-CAT normal range material $(E T P=1182 \mathrm{nmol} / \mathrm{L} \mathrm{min}$, $n=40$, PPP-reagent) established at the МСТH. The hyper-plasma group consisted of three persons with ETP values of $>2$ standard deviations (SD) of the mean from the same TGA-CAT normal range material as above. The FVIII:C (kIE/L) in the pooled plasma groups was 0.024 for hypo-, 0.55 for normal and 0.95 for hypercoagulable plasma.

Five test runs were conducted with each PPP-reagent where each run consisted of three RP, four hypo-plasmas, four normal plasmas and four hyper-plasmas in triplicate. Two test runs were scheduled each day to maximize RP and reagent usage.

\section{2 | Blood sampling and plasma preparation}

Pre-analytical variables were considered as previously reported. ${ }^{12}$ Peripheral venous blood was collected into BD Vacutainer ${ }^{\circledR}$ plastic tubes (Becton Dickinson, Franklin Lakes, NJ) with anticoagulant trisodium citrate $(0.109 \mathrm{~mol} / \mathrm{L}, \mathrm{pH} 7.4)$ using a $21 \mathrm{G}$ needle with a light tourniquet. PPP was prepared within 60 minutes of venipuncture by double centrifugation. The first centrifugation was at $2000 \mathrm{~g}$ for 10 minutes at RT, after which the supernatant was carefully isolated and then centrifuged a second time at $10000 \mathrm{~g}$ at RT for 10 minutes.

The doubly centrifuged plasma was pooled and aliquoted $(520 \mu \mathrm{L})$ in $0.5 \mathrm{~mL}$ cryotubes and rapidly frozen at $-80^{\circ} \mathrm{C}$. All samples were prepared at MCTH. Frozen plasma samples on dry ice were sent to the five other participating centres (Karolinska University Hospital, Solna, Sweden; Sahlgrenska University Hospital, Gothenburg, Sweden; Aarhus University Hospital, Skejby, Denmark; Rikshospitalet, Oslo, Norway; Helsinki University Central Hospital, Helsinki, Finland). All samples arrived frozen within 24 hours.

TABLE 1 Reference plasmas usage

\begin{tabular}{|c|c|c|c|}
\hline Reference plasma & \multicolumn{3}{|l|}{ Test samples } \\
\hline Siemens Control Plasma P (diluted $1+5$ ) & Dedicated normalization & & \\
\hline HemosIL Calibration Plasma & Normalization & Normalization & Normalization \\
\hline Affinity Protein S deficiency plasma & & & Dedicated normalization \\
\hline
\end{tabular}

Reference plasma (RP) used for normalization of data according to plasma types. Dedicated normalization is performed by a RP specifically chosen to normalize a certain type of plasma. 


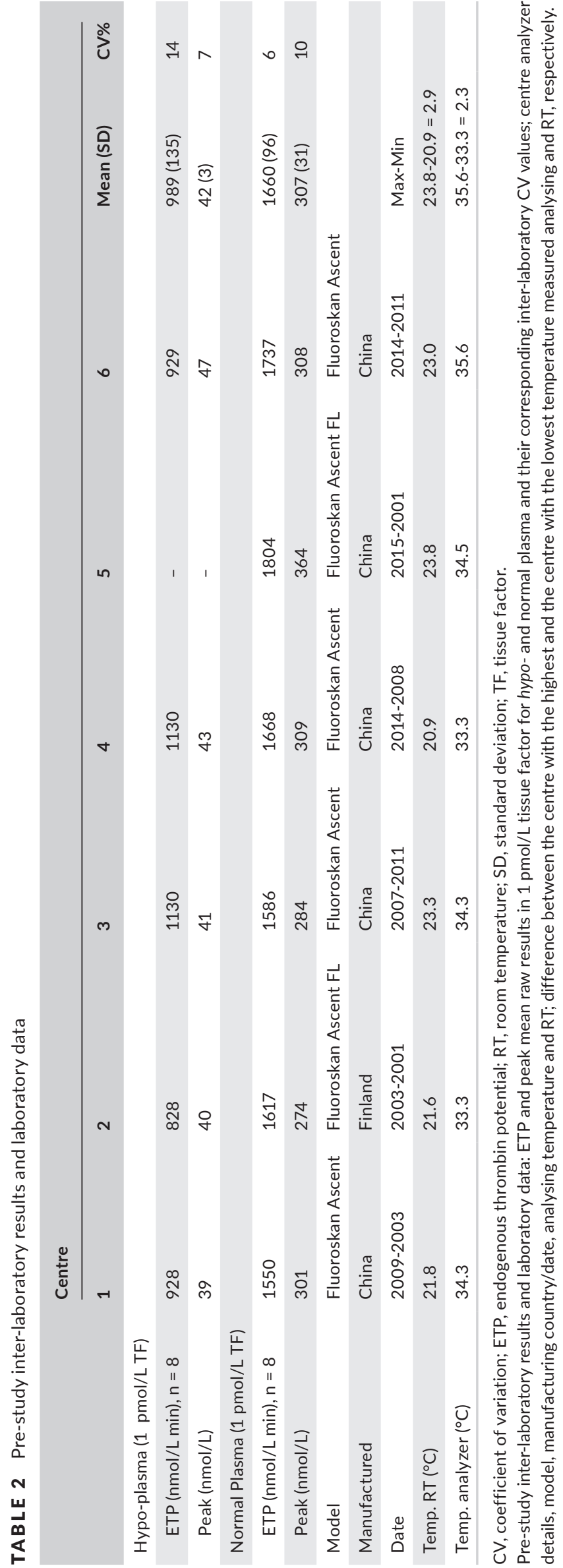

\section{3 | Reference lasma}

Several studies have shown that inter-centre variability can be decreased by normalization, that is, dividing all values with the result of a common RP run in each test. ${ }^{10,11}$

Three commercial lyophilized RPs were used for normalization of data. The rationale for testing three types of RP is that a previous study suggested a better inter-laboratory performance when using dedicated RPs, for example, a hypocoaguable RP for a hypocoagulable plasma sample and a hypercoagulable RP for a hypercoagulable sample (Table 1). ${ }^{11}$ Hypo-plasma results were normalized using Siemens Control Plasma P (diluted 1 + 5, Siemens RP; Siemens Healthcare Diagnostics Products $\mathrm{GmbH}$, Marburg, Germany), and hyper-plasma results were normalized applying protein $S$ deficiency plasma (PS-DP; Affinity RP; Affinity Biologicals Inc, Ancaster, ON, Canada). HemosIL Calibration Plasma (HemosIL RP; Instrumentation Laboratory, Bedford, MA) was chosen as a standard normalization plasma owing to the previous study by Dargaud et $\mathrm{al}^{10}$ where HemosIL RP was found to be the optimal RP compared to five other RPs.

\section{4 | TGA-CAT measurement}

TGA-CAT was measured using our detailed standardization laboratory protocol (Figure S1) based on the method described by Hemker et $\mathrm{al}^{13}$ with Thrombinoscope standardized reagents (Diagnostica Stago, Asnières, France). In short, $20 \mu \mathrm{L}$ PPP-reagent (1 or $5 \mathrm{pmol} / \mathrm{L}$ TF) and $20 \mu \mathrm{L}$ Thrombin Calibrator were manually pipetted into the wells of a round-bottom 96-well microtitre plate (Immulon 2HB; Thermo Scientific, Rochester, NY). Eighty microlitres of plasma were added to each PPP-reagent well and to its corresponding Thrombin Calibrator well. The plate was then placed in one of two models of Thermo Labsystems Fluoroskan Ascent readers, Diagnostica Stago for a 10 minutes, $37^{\circ} \mathrm{C}$ incubation. Following the incubation, $20 \mu \mathrm{L}$ of the starting reagent, FluCa-kit, was automatically dispensed into each well by the fluorimeter. The wavelengths of $390 \mathrm{~nm}$ (excitation) and $460 \mathrm{~nm}$ (emission) were used to detect the fluorescence intensity. Thrombin generation curves were calculated by a dedicated software program, Thrombinoscope (Diagnostica Stago) version: V5.0.0.742. The TGACAT software set-up and its version, samples, RPs and reagents were identical for all participating laboratories.

\section{5 | Statistical analysis}

The mean, standard deviation and the coefficient of variation (CV) (\%) were calculated for five TG parameters: endogenous thrombin potential (ETP), thrombin peak (Peak), lag time (LT), time to peak thrombin (ttPeak) and start tail time from which intra- and interassay results and inter-laboratory variability were obtained. Outliers were excluded using the outlier labelling method. ${ }^{14,15}$

To determine the level of agreement among the participating laboratories TGA-CAT results from all laboratories (up to $300 \mathrm{ob}$ servations) were plotted in Bland-Altman diagrams. Ninety-five per 
TABLE 3 Centre mean intra-assay CV (\%)

\begin{tabular}{llllll} 
& \multicolumn{2}{c}{ PPP-reagent LOW } & & \multicolumn{2}{c}{ PPP-reagent } \\
\cline { 2 - 3 } \cline { 5 - 6 } & ETP & Peak & & ETP & Peak \\
Hypo & 5.9 & 7.3 & & 5.6 & 5.8 \\
Normal & 6.4 & 5.2 & & 7.0 & 6.5 \\
Hyper & 3.5 & 3.4 & 3.5 & 3.1 \\
\hline
\end{tabular}

Mean intra-assay CV (\%) for ETP and peak values with hypo-, normal and hyper-samples and two triggering conditions: PPP-reagent LOW (1 pmol/L TF) and PPP-reagent (5 pmol/L TF).

$\mathrm{CV}$, coefficient of variation; ETP, endogenous thrombin potential; PPP, platelet-poor plasma.

cent of the observations within the acceptance margin $\pm 10 \%$ was set as acceptable. Statistical analysis was performed using IBM SPSS for Windows, Version 24.0. (IBM Corp., Armonk, NY).

\section{3 | RESULTS}

When presenting results, we have chosen to focus on ETP and Peak values which are often the main parameters referenced in clinical studies since they are considered most relevant from a biological perspective. $^{16,17}$

TGA-CAT mean EPT and Peak for the RPs should be added.

\section{1 | Pre-study results}

\subsection{1 | Intra-assay and inter-laboratory repeatability}

To evaluate the operator influence on results in the pre-study, one plate was divided into two parts. At one occasion, the inspector pipetted half of the wells and each centre's operator pipetted the other half. The intra-assay CV for ETP and Peak was $10 \%$ or lower for all participating centres. The pre-study inter-laboratory imprecision demonstrated CV results $10 \%$ or lower for ETP and Peak in both plasma types tested except for ETP in hypo-plasma, where the CV was $14 \%$ (Table 2).

\section{2 | Implementation study results}

\subsection{1 | Intra- and inter-assay repeatability}

The mean intra-assay CV was below 7.3\% for all of the hypo-, normal or hyper-plasma and TF concentrations tested (Table 3). Four of the six centres had inter-assay CVs of $10 \%$ or lower for all parameters (Table 4).

\subsection{2 | Inter-laboratory reproducibility}

The inter-laboratory CVs were $10 \%$ or below, under all test conditions, for all temporal data (LT, ST and ttPeak). For the ETP and Peak, a mean CV $<10 \%$ was obtained except in the case of hypo-plasma where only the Peak value in PPP-reagent had a CV below $10 \%$. The
TABLE 4 Centre inter-assay CV (\%)

\begin{tabular}{|c|c|c|c|c|c|}
\hline \multirow[b]{3}{*}{ Centre } & & \multicolumn{4}{|c|}{ Inter-assay CV (\%) } \\
\hline & & \multicolumn{2}{|c|}{ PPP-reagent LOW } & \multicolumn{2}{|c|}{ PPP-reagent } \\
\hline & & ETP & Peak & ETP & Peak \\
\hline 1 & Нypo & 7.7 & 7.9 & 5.9 & 5.4 \\
\hline 2 & & 20.3 & 19.3 & 29.8 & 24.8 \\
\hline 3 & & 10.1 & 10.2 & 6.1 & 7.7 \\
\hline 4 & & 8.1 & 8.2 & 5.2 & 7.3 \\
\hline 5 & & 13.8 & 15.7 & 0.7 & 1.9 \\
\hline 6 & & 8.0 & 7.3 & 8.6 & 8.5 \\
\hline 1 & Normal & 8.4 & 3.5 & 1.1 & 1.2 \\
\hline 2 & & 15.4 & 16.4 & 26.8 & 19.5 \\
\hline 3 & & 6.7 & 7.6 & 4.7 & 4.4 \\
\hline 4 & & 6.6 & 7.4 & 2.8 & 4.6 \\
\hline 5 & & 13.4 & 10.8 & 2.4 & 2.4 \\
\hline 6 & & 10.3 & 8.4 & 6.1 & 5.9 \\
\hline 1 & Hyper & 8.4 & 3.1 & 0.9 & 0.4 \\
\hline 2 & & 9.0 & 10.7 & 13.6 & 7.9 \\
\hline 3 & & 5.9 & 4.4 & 3.7 & 3.1 \\
\hline 4 & & 4.1 & 4.7 & 2.1 & 2.1 \\
\hline 5 & & 13.8 & 9.4 & 3.7 & 2.2 \\
\hline 6 & & 7.9 & 7.3 & 4.9 & 4.3 \\
\hline
\end{tabular}

CV, coefficent of variation; ETP, endogenous thrombin potential; PPP, platelet-poor plasma; TF, tissue factor.

Centre mean inter-assay CV\% for all variables in hypo-, normal and hyper-plasma samples with PPP-reagent LOW (1 pmol/L TF) and PPP-reagent (5 pmol/L TF).

other mean ETP and Peak values for hypo-plasma were between $12 \%$ and $14 \%$ (Table 5).

\subsubsection{Effect of normalization}

HemosIL RP was applied to normalize results over the entire measuring range. Siemens RP (dil. $1+5$ ) was applied for the hypo-samples and Affinity RP for the hyper-samples.

Normalization with HemosIL RP resulted in equal or lower CVs for all ETP and Peak values except for Hypo ETP/Peak and Hyper peak values with PPP-reagent LOW (Table 5, Figure 1). Normalization with Affinity RP resulted in lower CVs in three of four settings, while normalization with Siemens RP did not improve the CV in any of the four hypo-plasma cases.

\subsection{4 | Inter-laboratory agreement}

We considered $95 \%$ of the observations needed to be within a $\pm 10 \%$ acceptance level to be an adequate level of agreement measure for this study.

Results before and after normalization from the Bland-Altman plots are presented in Table 6 as the percentage of observations 


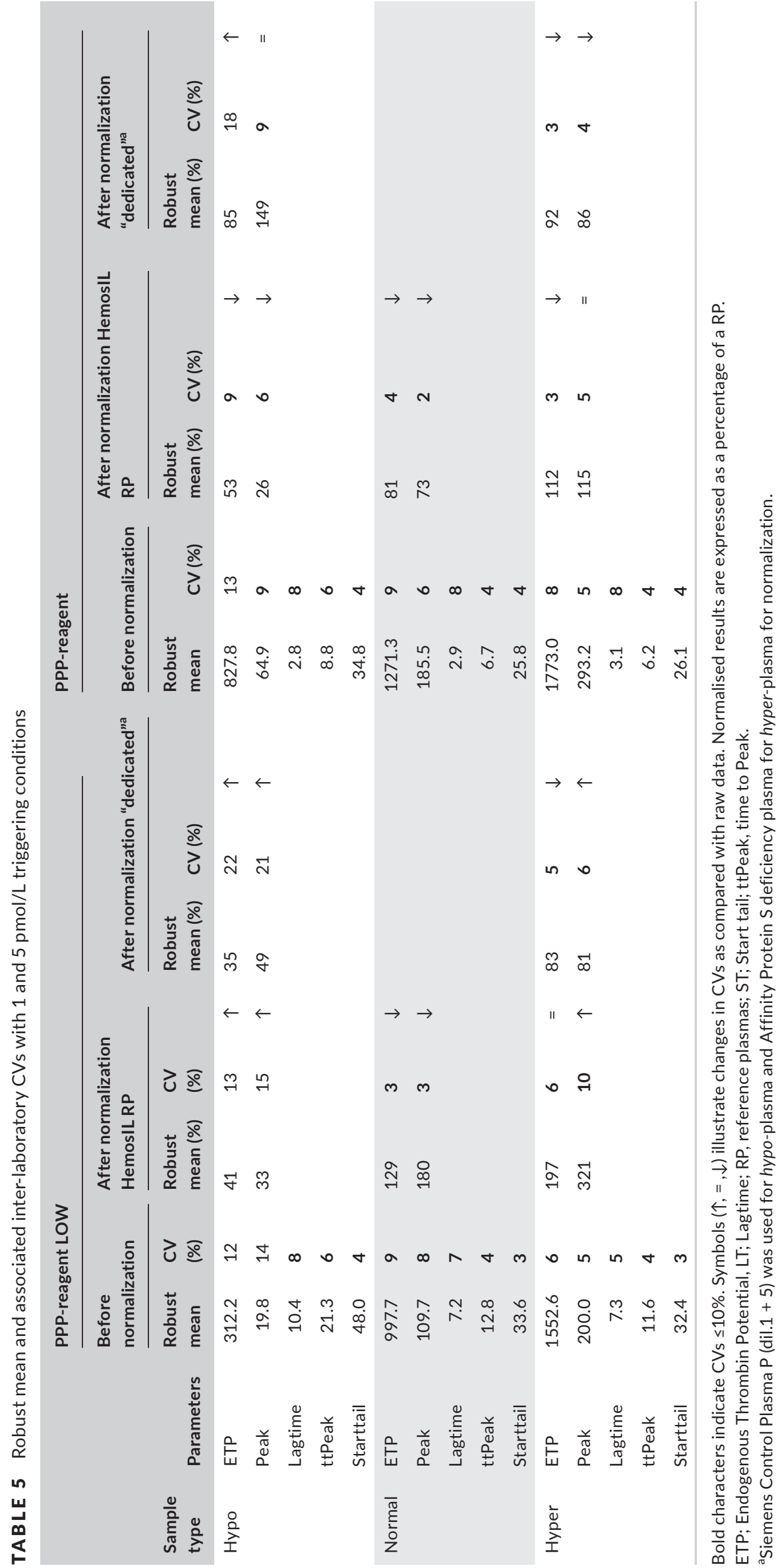




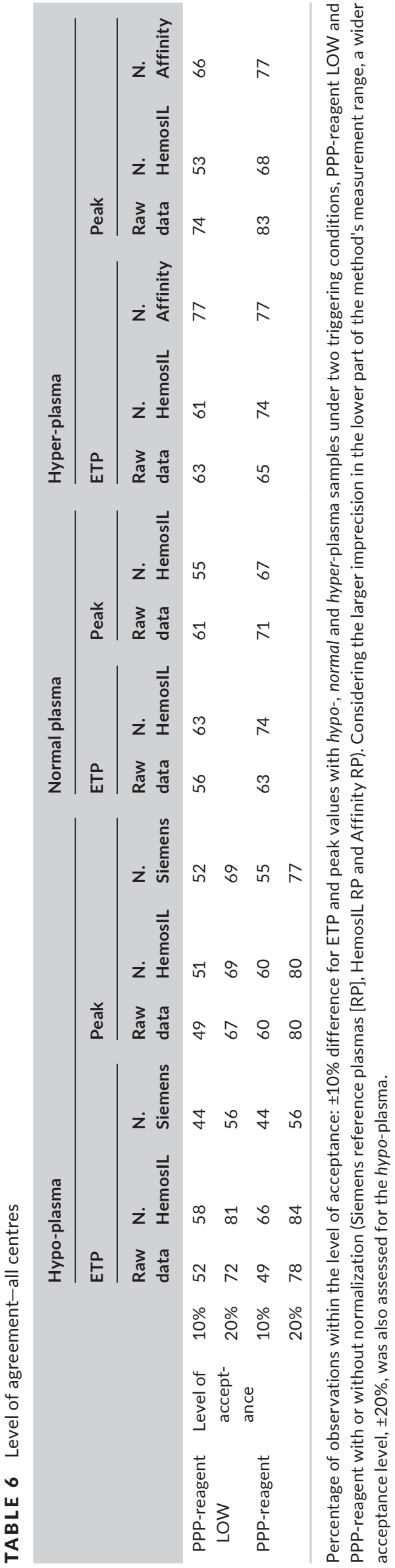

that met the level of acceptance, $\pm 10 \%$. Considering the larger imprecision in the lower part of the method's measurement range a wider acceptance level, $\pm 20 \%$ was also assessed for the hypoplasma. Widening the level of acceptance to $\pm 20 \%$ substantially increased the number of observations meeting the acceptance criteria.

The best level of agreement among ETP values was obtained by hyper-plasma in PPP-reagent. Sixty-five per cent of the observations were within $\pm 10 \%$ level of acceptance, and the observations improved to $77 \%$ after normalization with Affinity RP (Figure 2).

Normalization with RPs tended to affect the CV and level of agreement results equally. An exception was normalization with HemosIL RP which resulted in an equal or increased percentage of observations fulfilling the level of acceptance in all hypo-plasma measurements (Table 6) but not reductions in all the corresponding CVs (Table 5).

\section{4 | DISCUSSION}

In this study, we managed to obtain TGA-CAT results with quality in line with what routine laboratories analysis demand, $C V s \leq 10 \%,{ }^{11}$ by implementing our standardization concept, including a standardization protocol and synchronizing the level of quality and knowledge on included laboratories and their operators.

To improve the standardization level of the TGA-CAT method, we evaluated an elaborate standardization protocol, including a pre-study phase during which all participating centres' equipment and operators were controlled. We also tested the effect of normalization with three RPs, two of which are dedicated normalization plasmas.

The pre-study portion of our study was more important for obtaining lower variability results than expected. In order to increase compliance with the standardized protocol, it was made time point-based and built around a 50-minute countdown procedure. Moreover, all operators took part in the development of the protocol.

The intra-assay CVs were clearly decreased by introduction of the standardization protocol. This was most pronounced for the hypo-plasma (CV: from $14 \%$ to <8\%). In an article by Hemker et al, ${ }^{13}$ the biological variation for the TGA-CAT variable ETP was reported as $<5 \%$ for PPP and $<8 \%$ for PRP. Therefore, it is reasonable to aim for an intra-assay repeatability of $<10 \%$.

The most common discrepancy from the laboratory protocols was exceeding the time limits set in the 50-minute countdown schedule. None of the discrepancies, however, were considered to have major impact on the results.

Three of the six centres experienced unexpected difficulties during their 5-day analysing period. One centre's analyzer failed and was sent off site for maintenance in the middle of the testing period. Another centre had to use an operator who had not fully taken part in the pre-study operator check. The third centre experienced the problems of both centres noted above. Interestingly, exclusion 

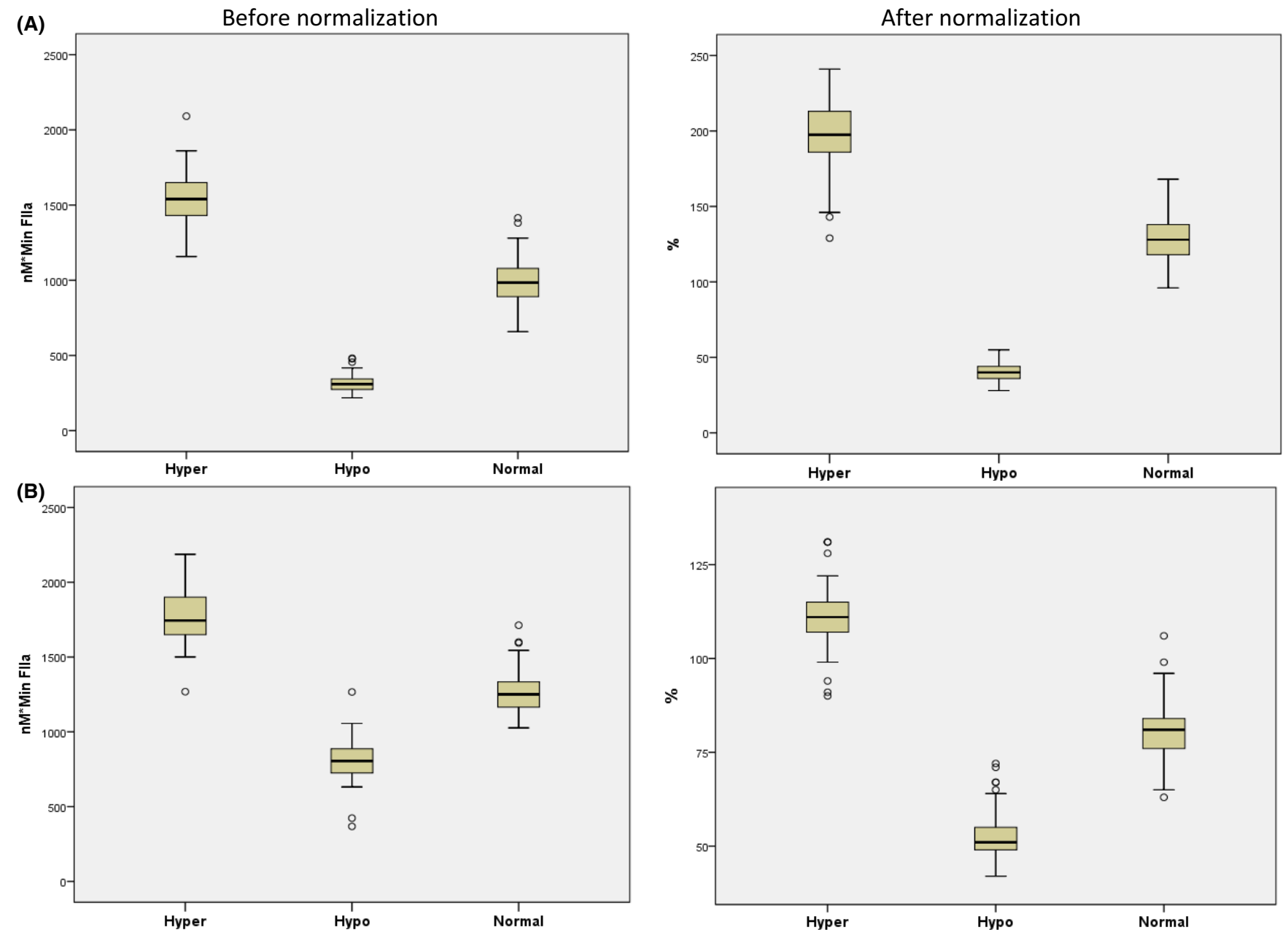

FIGURE 1 Thrombin generation was analysed in three different plasmas, hypo-, normal and hyper-plasma, with two trigger concentrations of TF, (A) $1 \mathrm{pmol} / \mathrm{L}$ and (B) $5 \mathrm{pmol} / \mathrm{L}$ tissue factor and ETP. Raw and after normalization results are displayed in the box plots. ETP, endogenous thrombin potential; TF, tissue factor

All centers - ETP, PPP-reagent, Hyper plasma

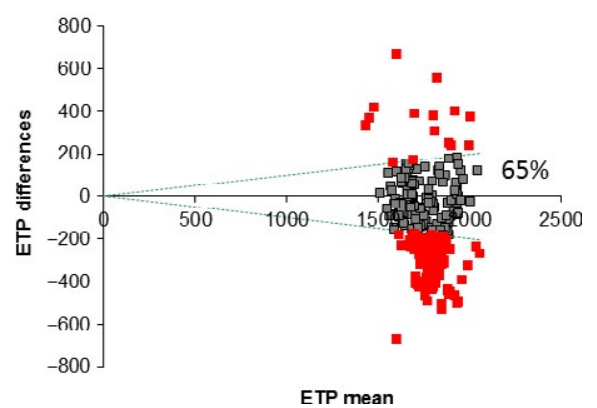

All centers - ETP normalized by Affinity RP, PPP-reagent, Hyper-plasma

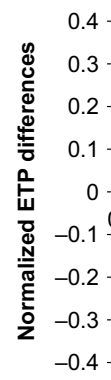

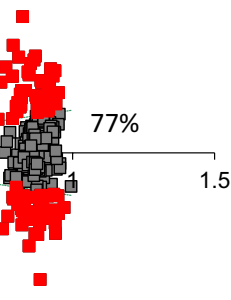

Normalized ETP mean

FIG URE 2 Results from all centres: ETP measurements in hyper-plasma analysed with PPP-reagent were plotted in a Bland-Altman plot. $65 \%$ of the observations were within the green dashed cone representing $\pm 10 \%$ level of acceptance. This level was improved to $77 \%$ after normalization with Affinity protein S deficiency plasma. ETP, endogenous thrombin potential; PPP, platelet-poor plasma

of results from the centres with unexpected difficulties yielded CVs $10 \%$ or lower for all samples under all test conditions. This fact indicates that when full compliance to our standardization protocol is met, inter-laboratory CV results of $10 \%$ or lower for all samples under all testing conditions, 1 and $5 \mathrm{pmol} / \mathrm{L}$, with hypo-, normal and hyper-plasma, are obtainable.

The Bland-Altman, or bias-plot, is used to describe systematic differences between methods. A limitation of the study design was 
that there was no reference method for comparison. Instead of being compared to an assigned value, the results from each centre were compared with the mean of all results.

Of the plasmas tested, analyses of hyper-plasma showed the best repeatability and, when comparing the TF-containing starting agents, analyses using PPP-reagent with the higher TF concentration ( $5 \mathrm{pmol} / \mathrm{L}$ ) had better repeatability than analyses using PPP-reagent LOW (1 pmol/L). This was in agreement with the pre-study measurements of intra-assay imprecision.

When evaluating the effect of normalization with the two dedicated plasmas in comparison with the "normal" normalization plasma, HemosIL RP, normalization with Affinity RP had the best effect. Normalization with this RP improved the repeatability in almost all settings, whereas normalization with Siemens RP rather decreased it. However, normalization with HemosIL RP improved the repeatability in all settings where PPP-reagent was used. However, it is plausible to believe that future studies evaluating dedicated normalization RPs will find PRs with better normalization abilities than ours. The sole purpose of the RP is to generate a denominator upon which to compare ratio values between assays. Thus, there is no need for RPs and test plasmas to be created under identical circumstances.

In a recent article by Hemker, ${ }^{19}$ three substantial obstacles for the method to overcome are mentioned, of which thermostability is the most significant. In our pre-study, we observed a $2.3^{\circ} \mathrm{C}$ difference between the centre with the highest and the centre with the lowest measured analysing temperature (Table 1). The pre-study TGA-CAT results, ETP and peak with hypo- and normal plasma, did not correlate well with the analysing temperature measurements which may be explained by the small number of observations. However, a $2.3^{\circ} \mathrm{C}$ difference is associated with $10 \%-20 \%$ higher ETP and Peak values according to tests conducted at MCTH (results not shown).

The difference in RT among the laboratories was $2.9^{\circ} \mathrm{C}$. The correlation between RT and analysing temperature is $r=0.693$ which may explain some of the differences in analysing temperatures for the six centres. Tests at MCTH showed that an increase of $3^{\circ} \mathrm{C}$ in $\mathrm{RT}$ generates a rise in analysing temperature of close to $1^{\circ} \mathrm{C}$. This is equivalent with up to a $10 \%$ increase in ETP and Peak values. Based on our analysing temperature observations, we believe there is an improvement potential to our standardization concept by synchronizing the analysing temperatures of all included laboratories through manipulating the software temperature set-up. We estimate that the temperature gap of $2.3^{\circ} \mathrm{C}$ could be narrowed down to $1^{\circ} \mathrm{C}$, and we consider this temperature control issue would be interesting to evaluate in future standardization studies.

One of the more important instructions in the protocol is to start the analyzer at least one hour before the start of incubation. Measurements at MCTH have shown that it takes about 45 minutes for the Fluoroskan Ascent to reach its analysing temperature of $37^{\circ} \mathrm{C}$, a temperature that, according to the Thrombinoscope software, is reached within only 10 minutes. This step together with the compliance increasing features of our standardization concept probably contributed most to the results of this study.
To conclude, our standardization study produced TGA-CAT results as robust as standard coagulation assays used in the routine laboratories where all results under all testing conditions showed CVs of $10 \%$ or lower except for ETP (12\%) and peak (14\%) in hypoplasma with $1 \mathrm{pmol} / \mathrm{L}$ TF. Normalization with HemosIL RP decreased the inter-laboratory imprecision in most plasma samples. Normalization with a dedicated normalization plasma (Affinity RP, protein $\mathrm{S}$ deficiency plasma) performed even better than HemosIL $\mathrm{RP}$ in the hypercoagulable measurement range. However, the dedicated normalization plasma Siemens RP did not perform better than HemosIL RP in that range. Thus, normalization with Affinity RP could be considered when analysing plasma samples from populations where hypercoagulability is known or suspected. In populations with low or unknown coagulability, normalization with HemosIL RP could be considered.

\section{ACKNOWLEDGEMENTS}

This study was supported by funds from Baxalta, Region Skåne and Lund University (Regional funds and ALF). We would like to thank Nida Mahmoud Hourani Soutari, BS, Nina Schultz, MD, Stine Bjørnsen, BS, Vivi Bo Mogensen, BS, Mai Stenulm Therkelsen, BS for performing the experiments and contributing to the development of the laboratory protocol. Ole Halfdan Larsen has received speaker and consultant fees from Shire and speaker fees from Baxalta. The other authors stated that they had no interests which might be perceived as posing a conflict or bias.

\section{DISCLOSURES}

Ole Halfdan Larsen has recieved speaker and consultant fees from Shire, and speaker fees from Baxalta. The other authors stated that they had no interests which might be percieved as a conflict or bias.

\section{AUTHORS CONTRIBUTIONS}

Marcus Ljungkvist designed and coordinated the study, performed the experiments and wrote the manuscript. Eva Zetterberg designed the study and wrote the manuscript. Erik Berntorp designed the study and wrote the manuscript. Karin Strandberg designed the study and wrote the manuscript. Roza Chaireti wrote the manuscript, performed the experiments and provided laboratory resources. Pål André Holme wrote the manuscript and provided laboratory resources. Ole Halfdan Larsen wrote the manuscript and provided laboratory resources. Riitta Lassila wrote the manuscript and provided laboratory resources. Annukka Jouppila wrote the manuscript and performed the experiments. Timea Szanto wrote the manuscript and performed the experiments.

\section{ORCID}

Marcus Ljungkvist (iD https://orcid.org/0000-0001-9855-6485

Erik Berntorp (iD https://orcid.org/0000-0002-1337-7195 
Roza Chaireti (iD https://orcid.org/0000-0002-9069-1903

Timea Szanto iD https://orcid.org/0000-0001-7006-6564

Eva Zetterberg (iD https://orcid.org/0000-0003-1775-1727

\section{REFERENCES}

1. Nair SC, Dargaud Y, Chitlur M, Srivastava A. Tests of global haemostasis and their applications in bleeding disorders. Haemophilia. 2010;16(Suppl 5):85-92.

2. Trossaert $M$, Regnault $V$, Sigaud $M$, Boisseau $P$, Fressinaud $E$, Lecompte T. Mild hemophilia A with factor VIII assay discrepancy: using thrombin generation assay to assess the bleeding phenotype. J Thromb Haemost. 2008;6(3):486-493.

3. Dargaud $Y$, Luddington R, Baglin TP. Elimination of contact factor activation improves measurement of platelet-dependent thrombin generation by calibrated automated thrombography at low-concentration tissue factor. J Thromb Haemost. 2006:4(5):1160-1161.

4. Lecompte T, Wahl D, Perret-Guillaume C, Hemker HC, Lacolley P, Regnault $\mathrm{V}$. Hypercoagulability resulting from opposite effects of lupus anticoagulants is associated strongly with thrombotic risk. Haematologica. 2007;92(5):714-715.

5. Freyburger G, Macouillard G, Labrouche S, Sztark F. Coagulation parameters in patients receiving dabigatran etexilate or rivaroxaban: two observational studies in patients undergoing total hip or total knee replacement. Thromb Res. 2011;127(5):457-465.

6. Hacquard M, Perrin J, Lelievre N, Vigneron C, Lecompte T. Inter-individual variability of effect of 7 low molecular weight antithrombin-dependent anticoagulants studied in vitro with calibrated automated thrombography. Thromb Res. 2011;127(1):29-34.

7. Loeffen R, Kleinegris MC, Loubele ST, et al. Preanalytic variables of thrombin generation: towards a standard procedure and validation of the method. J Thromb Haemost. 2012;10(12):2544-2554.

8. Rodgers SE, Wong A, Gopal RD, Dale BJ, Duncan EM, McRae SJ. Evaluation of pre-analytical variables in a commercial thrombin generation assay. Thromb Res. 2014;134(1):160-164.

9. Dargaud Y, Wolberg AS, Luddington R, et al. Evaluation of a standardized protocol for thrombin generation measurement using the calibrated automated thrombogram: an international multicentre study. Thromb Res. 2012;130(6):929-934.

10. Dargaud $\mathrm{Y}$, Luddington $\mathrm{R}$, Gray $\mathrm{E}$, et al. Standardisation of thrombin generation test-which reference plasma for TGT? An international multicentre study. Thromb Res. 2010;125(4):353-356.

11. Perrin J, Depasse F, Lecompte T,French-speaking CATg, under the aegis of G, French-speaking CATg, French-speaking CATgaiFuos.
Large external quality assessment survey on thrombin generation with CAT: further evidence for the usefulness of normalisation with an external reference plasma. Thromb Res. 2015;136(1):125-130.

12. Dargaud Y, Wolberg AS, Gray E, Negrier C, Hemker HC, Subcommittee on Factor Viii FIX, Rare Coagulation D. Proposal for standardized preanalytical and analytical conditions for measuring thrombin generation in hemophilia: communication from the SSC of the ISTH. J Thromb Haemost. 2017;15(8):1704-1707.

13. Hemker HC, Giesen P, Al Dieri R, et al. Calibrated automated thrombin generation measurement in clotting plasma. Pathophysiol Haemost Thromb. 2003;33(1):4-15.

14. Hoaglin DC, Iglewicz B, Tukey JW. Performance of some resistant rules for outlier labeling. J Am Stat Assoc. 1986;81:991-999.

15. Hoaglin DC, Iglewicz B. Fine tuning some resistant rules for outlier labeling. J Am Stat Assoc. 1987;82:1147-1149.

16. Dargaud Y, Lienhart A, Negrier C. Prospective assessment of thrombin generation test for dose monitoring of bypassing therapy in hemophilia patients with inhibitors undergoing elective surgery. Blood. 2010;116(25):5734-5737.

17. Salvagno GL, Astermark J, Lippi G, et al. Thrombin generation assay: a useful routine check-up tool in the management of patients with haemophilia? Haemophilia. 2009;15(1):290-296.

18. Dargaud $Y$, Beguin $S$, Lienhart $A$, et al. Evaluation of thrombin generating capacity in plasma from patients with haemophilia A and $B$. Thromb Haemost. 2005;93(3):475-480.

19. Hemker HC. The application of thrombin generation in real life clinical situations. Thromb Res. 2015;136(1):3-4

\section{SUPPORTING INFORMATION}

Additional supporting information may be found online in the Supporting Information section at the end of the article.

How to cite this article: Ljungkvist M, Strandberg K, Berntorp E, et al. Evaluation of a standardized protocol for thrombin generation using the calibrated automated thrombogram: A Nordic study. Haemophilia. 2019;25:334342. https://doi.org/10.1111/hae.13640 